\title{
Models for Monolayers Adsorbed on a Square Substrate
}

\author{
Ofer Biham, Lee-Wen Chen, and Gianfranco Vidali
}

\author{
Department of Physics \\ Syracuse University \\ Syracuse, NY 13244
}




\begin{abstract}
Motivated by recent experimental studies of $\mathrm{Hg}$ and $\mathrm{Pb}$ monolayers on $\mathrm{Cu}(001)$ we introduce a zero temperature model of a monolayer adsorbed on a square substrate. Lennard-Jones potentials are used to describe the interaction between pairs of adlayer-adlayer and adlayer-substrate atoms. We study a special case in which the monolayer atoms form a perfect square structure and the lattice constant, position and orientation with respect to the substrate can vary to minimize the energy. We introduce a rule based on the Farey tree construction to generate systematically the most energetically favored phases and use it to calculate the phase diagram in this model.
\end{abstract}




\section{Introduction}

The study of ordered phases of films of one layer or less on metal substrates is an active topic of research in two-dimensional physics [1]. Due to improved film deposition and detection techniques, it is now possible to investigate more systems and with better resolution than has been possible before [2]. Experimental studies of ordered overlayers show a great variety of commensurate and incommensurate

phases [3, 4]. Here we define a commensurate phase as an ordered structure for which it is possible to find a primitive cell with basis vectors which are linear combinations of lattice vectors of the substrate. If this primitive cell (supercell) is larger than the overlayer unit cell and contains several atoms, then we will call this a high-order commensurate structure. When the number of atoms in this cell goes to infinity, the high-order commensurate structure becomes incommensurate.

With some exceptions, structures of strongly chemisorbed systems are either fully commensurate (every adatom is in registry with the substrate lattice) or incommensurate phases; while high-order commensurate phases are less often seen among systems in which strong adatom localization occurs. Because of the localized nature of the adsorption and the poor understanding of the interaction potentials, theoretical methods to describe the structure and phase boundaries of these layers have relied by and large on lattice gas models and the parametrization of interaction energies [5, 6]. 
In the opposite limit, when incommensurate structures are formed, other approaches have been tried. Calculations by Novaco and McTague [7] and Shiba [8] showed that orientational epitaxy might arise in an incommensurate layer adsorbed on a substrate; this effect has been observed for rare-gas monolayers adsorbed on graphite and for certain alkali monolayers on metal substrates.

In this work we introduce a model that describes an ordered layer with square symmetry adsorbed on a square substrate. This model is used to compute the zero temperature phase diagram for this system.

\section{Experimental Background}

Recently, high-order commensurate structures have been detected for $\mathrm{Hg}$ and $\mathrm{Pb}$ adsorbed on $\mathrm{Cu}(001)$ [9, 10]. In addition to the fully commensurate $C(2 \times 2)$ phase, two high order commensurate square phases have been observed and studied in details: the $C(4 \times 4)$ phase $(\mathrm{Hg}$ on $\mathrm{Cu})$ and the $(5 \times 5) R \tan ^{-1}(3 / 4)$ phase $(\mathrm{Pb}$ on $\mathrm{Cu}$ ). Only one of the atoms of the supercell is in the ideal registry position, while all the others sit in lesser energetically favorable locations. The experimental results indicate that in these phases the adlayer forms an almost perfect square lattice with only small local relaxations caused by the substrate potential.

Surprisingly, these high-order commensurate phases possess square symmetry. We recall that for an unsupported layer with Lennard-Jones pairwise interactions 
the most stable structure has hexagonal symmetry [11]. It is then clear that these high-order commensurate square structures must be stabilized by substrate forces and/or by more complicated interactions, such as three-body forces or angular dependent, covalent-like interactions [5].

\section{Classification of Square Phases}

We have developed a system, based on the two dimensional analog of the Farey tree of rational numbers [12], to classify all the commensurate square phases on a square substrate. Consider a square monolayer on a square substrate such as the $(5 \times 5) R \tan ^{-1}(3 / 4)$ phase (Fig. 1). The unit cell of the monolayer is a square, which is typically rotated with respect to the substrate main symmetry directions. To characterize the structure we pick a side of the square and project it on the $\mathrm{x}$ and $\mathrm{y}$ directions. Both projections are integer multiples of the substrate lattice constant, which will be given by the integers $p_{x}$ and $p_{y}$ respectively. The linear size of the supercell, in terms of the monolayer lattice constant, is given by $q$. Therefore, one can identify each square phase by the pair of rational numbers

$$
\left(\frac{p_{x}}{q}, \frac{p_{y}}{q}\right)
$$

Using this observation we propose a systematic hierarchical procedure to generate a class of commensurate square phases. The advantage of this method is that it generates a sequence of phases from the most stable and lowest order ones up to 
the least energetically favorable.

The list of phases is constructed by starting with a set of four lowest order phases (furthest corners in Table 1). Then between each pair of neighboring phases one adds a new phase for which the new $p_{x}, p_{y}, q$ are the sums of the corresponding quantities in the neighboring phases. The four new phases are located at each side of the square (midway between corners in Table 1). A new phase is also introduced in the center, using as input the two phases on one of the diagonals. Here one should choose the diagonal that includes a phase which belongs to a lower generation.

One can continue this procedure by breaking the new diagram into four separate smaller squares and repeating the procedure for each one of them. The main advantage of this procedure is that it generates phases of low order first (which are most likely to be stable) while higher order phases appear in later generations. This construction should provide a useful guide for the experimentalist willing to explore interesting yet undetected structures which are in the vicinity of experimentally observed ones.

\section{The Model}

In order to obtain a better understanding of which adlayer structures one should expect to find on a square substrate, we have studied a zero temperature model and

calculated its phase diagram. In particular we consider a two dimensional layer of 
atoms with Lennard-Jones interactions, subject to a periodic underlying potential due to interactions with the substrate. Since the Lennard-Jones interaction is of long range, each atom in the overlayer interacts with all the other atoms of the overlayer and the substrate. The lattice constant, orientation and position with respect to the substrate can all be changed to minimize the energy. Since the square phases detected experimentally seem to show little relaxation (and this is born out by a mean-field type of calculation [11]), one can consider that the adlayer atoms are constrained to a perfect square structure.

In this simplified case one can use results for lattice sums to add the adlayersubstrate interactions into an effective potential. The energy will then be:

$$
E=\sum_{m, n}^{\prime} V_{L J}\left(\vec{r}_{m}-\vec{r}_{n}\right)+\sum_{n} V_{S}\left(\vec{r}_{n}\right)
$$

where

$$
V_{L J}(\vec{r})=4 \varepsilon_{a d}\left[\left(\frac{\sigma_{a d}}{|\vec{r}|}\right)^{12}-\left(\frac{\sigma_{a d}}{|\vec{r}|}\right)^{6}\right]
$$

is the Lennard-Jones potential between the adlayer atoms, $V_{S}$ is the underlying substrate potential and $\vec{r}_{n}=\left(x_{n}, y_{n}\right)$ is the position of the nth adlayer atom. The substrate potential is given by:

$$
V_{S}\left(\vec{r}_{n}\right)=A_{0}+\sum_{\vec{k}} A_{\vec{k}} e^{\vec{k} \cdot \vec{r}_{n}}
$$

where the sum runs over all the reciprocal lattice vectors of the substrate. The 
Fourier coefficients are given by [13]

$$
A_{0}=8 \pi \varepsilon \sigma^{2}\left[\frac{2}{5}\left(\frac{\sigma}{z_{0}}\right)^{10}-\left(\frac{\sigma}{z_{0}}\right)^{4}\right]
$$

and

$$
A_{\vec{k}}=\frac{8 \pi \varepsilon \sigma^{12}}{30 a_{s b}^{2}}\left(\frac{k}{2 z_{0}}\right)^{5} K_{5}\left(k z_{0}\right)-\frac{8 \pi \varepsilon \sigma^{6}}{a_{s b}^{2}}\left(\frac{k}{2 z_{0}}\right)^{2} K_{2}\left(k z_{0}\right)
$$

where $k=|\vec{k}|, K_{p / q}$ is a modified Bessel function of order $\mathrm{p} / \mathrm{q}, z_{0}$ is the distance between the adlayer and substrate and $a_{s b}=\left(2 C_{12} / C_{6}\right)^{1 / 6} \cdot \sigma_{s b}$ is the lattice constant of the substrate where $C_{n}=\frac{1}{2} \sum_{i, j}^{\prime} 1 /\left(i^{2}+j^{2}\right)^{n / 2}$. Here, the parameters $\sigma$ and $\varepsilon$ for the adlayer-substrate interaction are given by $\sigma=\left(\sigma_{a d}+\sigma_{s b}\right) / 2$ and $\varepsilon=\sqrt{\varepsilon_{a d} \varepsilon_{s b}}$. For simplicity we choose $\varepsilon_{s b}=1$ and $\sigma_{s b}=1$. The distance $z_{0}=\sigma$ between the adlayer and the substrate is then determined so that the zeroth order term, $A_{0}$, in the potential is minimized. Note that we assume here that the distance $z_{0}$ between the monolayer and the substrate is the same for all adlayer atoms, although in reality these heights vary $(0.1-0.2 \AA)$ [14] and depend on the position of the atom with respect to the substrate.

To study the phase diagram we first choose a structure and then calculate the energy per atom. We compare these energies for a set of commensurate and incommensurate square phases and obtain the phase diagram for the physically interesting regime of $1<\sigma_{a d}<2$ (Fig. 2). The parameters in the phase diagram are $\sigma_{a d}$, and $1 / \sqrt{\varepsilon_{a d}}$, the latter being proportional to the strength of the underlying substrate potential. We normalize the lattice constant of the adlayer in units of 
the substrate periodicity and define $a=a_{a d} / a_{s b}$. The phase diagram contains both commensurate and incommensurate square phases. Each commensurate phase is stable in a tongue of a finite width, in which $a=\sqrt{p_{x}^{2}+p_{y}^{2}} / q$, while incommensurate phases exist in the gaps between these tongues. In Fig. 2 one can see that the tongues start very narrow and as the parameter $1 / \sqrt{\varepsilon_{a d}}$ increases they become broader until they intersect in triple points. Above the triple point there is a first order transition line between the two tongues, while all the incommensurate phases between them disappear. The phase diagram is asymmetric and this is due to the hard core nature of the Lennard-Jones potential. The adlayer phase can expand considerably in order to gain energy from the underlying substrate potential but it cannot contract as much due to the hard core repulsion. Also note that the high order tongues are extremely thin. This is due to the asymptotic properties of the modified Bessel functions, which appear in the Fourier coefficients $A_{k}$. For high order harmonics of the potential the amplitudes decay exponentially, or $A_{k} \propto e^{-z_{0} k}$. Thus, the energy gain of high order commensurate phases becomes rapidly very small as the order of commensuration increases.

Finally, we have calculated the phase diagram with the inclusion of (incommensurate) hexagonal phases; in this case, most of the square phases are washed out, except for the fully commensurate ones. This is to be expected, since high order phases appear for weak interactions with the substrate (lower part of Fig. 2) and 
the energy gain for commensuration is small.

\section{Conclusion}

In summary, we have studied a simple model for the energetics of square monolayer phases on a square substrate. To study the phase diagram we introduced a simple classification that allowed us to find the most energetically favored phases. We find that when only square phases are considered, each one of them has a tongue in which it is stable. The $C(4 \times 4)$ phase $(\mathrm{Hg}$ on $\mathrm{Cu})$ and the $(5 \times 5) R \tan ^{-1}(3 / 4)$ phase $(\mathrm{Pb}$ on $\mathrm{Cu}$ ) belong to very narrow tongues (not shown in Fig.2) which intersect the $\sigma_{a d}$ axis at 1.265 and 1.25 , respectively. The fact that hexagonal phases win out (in our calculation) might be due to a variety of factors, including the fact that the observed phases are metastable and the actual interactions might be more complicated than the ones used in this study.

One of us (G.V.) would like to acknowledge partial support from NSF grant 8802512 . 


\section{References}

[1] N.D. Shrimpton, M.W. Cole, and W.A. Steele, in Phase Transitions in Surface Films, Ed. H. Taub, G. Torzo, H. Lauter, and S.C. Fain, (Plenum, New York, 1991); R.D. Dhiel, ibid.

[2] W.N. Unertl, Comments Cond. Mat. Phys., 12, 289 (1986).

[3] P. Bak, Rep. Prog. Phys., 45, 587 (1982); J. Villain and M.B. Gordon, Surface Science, 125, 1 (1983).

[4] L. Roelofs, Appl. Surf. Sci. 11/12, 425 (1982); E. Bauer, in Structure and Dynamics of Surfaces, Ed. W. Schommers and P. von Bleckenhagen, Topics in Current Physics, Volume 43 (Springer Verlag, 1987), p.115.

[5] T.L. Einstein, in Chemistry and Physics of Solid Surfaces, R. Vanselow and R.F. Howe Eds., (Springer Verlag, 1988), p.307.

[6] K. Binder and D.P. Landau, Advances in Chemical Physics, Ed. K.P. Lawley (John Wiley \& Sons, 1989) p. 91.

[7] J.P. McTague and A.D. Novaco, Phys. Rev. B19, 5299 (1979).

[8] H. Shiba, J. Phys. Soc. of Japan 46, 1852 (1979);48, 211 (1980).

[9] W. Li, J. Lin, M. Karimi, P.A. Dowben and G. Vidali, Phys. Rev. B45, 3708 (1992). 
[10] W. Li, J. Lin, M. Karimi, C. Moses and G. Vidali, Applied Surface Science 48/49, 160 (1991).

[11] O.Biham, W.Chen, L.-W. Chen, and G.Vidali, in preparation.

[12] See e.g., I. Niven and H.S. Zuckerman, An Introduction to the Theory of Numbers, John Wiley \& Sons, 1966.

[13] W.A. Steele, Surface Science 36, 317 (1973).

[14] W. Li, J. Lin, M. Karimi and G. Vidali, J. Vac. Sci. Tech. A9, 1707 (1991). 


\section{$\underline{\text { Figure Captions }}$}

Figure 1: The $(5 \times 5) R \tan ^{-1}(3 / 4)$ phase that appears in $\mathrm{Pb}$ on $\mathrm{Cu}(001)$. Empty circles represent substrate atoms, while full circles represent adlayer atoms.

Figure 2: Phase diagram (at $0 \mathrm{~K}$ ) of two dimensional homogeneous square phases on a square substrate, with Lennard-Jones interactions. Note the clear asymmetric shape, which is due to the asymmetry of the Lennard-Jones potential. It includes three fully commensurate square phases: $1 \times 1$ (or $(0 / 1,1 / 1)), C(2 \times 2)$ (or $(1 / 1,1 / 1))$ and $2 \times 2$ (or $(0 / 1,2 / 1))$. It also includes a few examples of the infinitely many high order commensurate square phases (from left to right): $[1 / 2,2 / 2]$, $\{3 / 5,6 / 5\},[0 / 2,3 / 2],[1 / 2,3 / 2]$, and $\{3 / 4,7 / 4\}$.

\section{Table Caption}

Table 1: A classification for square monolayer phases on a square substrate. In this hierarchical classification, the lowest order phases, which are typically the most stable, appear first, while higher order phases appear in later generations. Generations are distinguished by different symbols: ( ), [ ], \langle\rangle , and \{\} for generations $1,2,3$ and 4, respectively. For example, the experimentally observed high order commensurate square phases $C(4 \times 4)$ and $(5 \times 5) R \tan ^{-1}(3 / 4)$ appear here as $\{2 / 5,6 / 5\}$ and $\{3 / 4,4 / 4\}$ respectively. 

$\left(\frac{0}{1}, \frac{1}{1}\right)$
$\left[\frac{0}{2}, \frac{3}{2}\right]$
$\left(\frac{0}{1}, \frac{2}{1}\right)$
$\left\langle\frac{1}{3}, \frac{3}{3}\right\rangle$
$\left\langle\frac{1}{3}, \frac{4}{3}\right\rangle$
$\left\{\frac{2}{5}, \frac{6}{5}\right\}$
$\left[\frac{1}{2}, \frac{2}{2}\right]$
$\left\langle\frac{2}{4}, \frac{5}{4}\right\rangle$
$\left[\frac{1}{2}, \frac{3}{2}\right]$
$\left[\frac{1}{2}, \frac{4}{2}\right]$
$\left\langle\frac{2}{3}, \frac{3}{3}\right\rangle \quad\left\{\frac{4}{6}, \frac{7}{6}\right\} \quad\left\langle\frac{2}{3}, \frac{4}{3}\right\rangle$
$\left\{\frac{3}{4}, \frac{4}{4}\right\} \quad\left\{\frac{3}{4}, \frac{5}{4}\right\} \quad\left\{\frac{5}{6}, \frac{8}{6}\right\}$
$\left(\frac{1}{1}, \frac{1}{1}\right) \quad\left\{\frac{4}{4}, \frac{5}{4}\right\} \quad\left\langle\frac{3}{3}, \frac{4}{3}\right\rangle$
$\left[\frac{2}{2}, \frac{3}{2}\right]$
$\left(\frac{1}{1}, \frac{2}{1}\right)$ 